\title{
Molecular Orientation in Deformed Bimodal Networks. 1. Theory
}

\section{Bahar* and B. Eriman}

Polymer Research Center and School of Engineering, Bogazici University, and TUBITAK Advanced Polymeric Materials Research Center, Bebek 80815, Istanbul, Turkey

\section{Bokobza and L. Monnerie}

Laboratoire de Physico-Chimie Structurale et Macromoléculaire, ESPCI, 10 rue Vauquelin, 75231 Paris Cedex 05, France

Received March 14, 1994; Revised Manuscript Received September 7, $1994^{\otimes}$

\begin{abstract}
Expressions for the molecular orientation of long and short chains in bimodal networks, $S_{\mathrm{L}}$ and $S_{\mathrm{S}}$, are derived based on the theory developed by Kloczkowski et al. (Macromolecules 1991, 24, 3266) for regular bimodal phantom networks and compared with those $\left(S_{\mathrm{L}}{ }^{\circ}\right.$ and $S_{\mathrm{S}}{ }^{\circ}$ ) occurring in corresponding unimodal networks. The changes in segmental orientation of the respective chains in bimodal networks relative to their unimodal counterparts, expressed as the ratio $S_{\mathrm{L}} / S_{\mathrm{L}}{ }^{\circ}$ or $S_{\mathrm{S}} / S_{\mathrm{S}}{ }^{\circ}$, are shown to be independent of macroscopic strain and intrinsic chain configurational characteristics but depend essentially on network topology and composition. The latter are accounted for by two variables: the ratio $\xi$ of the molecular weights of short chains to that of long chains, and the number $\phi_{\mathrm{S}}$ and $\phi_{\mathrm{L}}$ of short and long chains at each junction. Results of the formulation show that the long (short) chains in the bimodal network orient more (less) than those in the corresponding unimodal network. These differences in the orientation behavior of the two chains arise from differences in fluctuations of chain dimensions that, in turn, affect the microscopic strain of each component. Predictions of the theory are compared in the following paper with results of Fourier transform infrared measurements on well-defined poly(dimethylsiloxane) networks.
\end{abstract}

\section{Introduction}

Mechanical properties of bimodal poly(dimethylsiloxane) (PDMS) networks consisting of short and relatively long chains were experimentally studied by Mark and co-workers ${ }^{1}$ to understand the rubber elasticity behavior of these systems. Such networks were found to be unusually tough. They exhibit values of the modulus which increase very substantially at high elongations, thus giving unusually large values of the ultimate strength. This improvement in mechanical properties was attributed to the limited extensibility of the short chains.

The theoretical analysis of bimodal networks, on the other hand, was first performed by Higgs and Ball. ${ }^{2}$ These networks are composed of two types of chains, conveniently referred to as short and long chains, differing either in their molecular weight or in their chemical structure, thus obeying two distinct probability distribution functions for their end-to-end separations. The original theoretical approach, based on Gaussian phantom network chains for both components, was essentially developed for random bimodal networks with a random number of short or long chains connected at a given junction. Later, Kloczkowski et al. $^{3}$ considered the statistical mechanics of regular bimodal networks, which, by definition, have a fixed number $\phi_{\mathrm{S}}$ and $\phi_{\mathrm{L}}$ of short and long chains, respectively, at every junction and hence lend themselves to analytical solutions. Closed-form expressions were derived therein for the fluctuations and correlations in the positions of the junctions. The validity of the affine deformation of mean chain dimensions in bimodal networks, irrespective of the lengths or structural characteristics of the two types of chains, was verified recently by computer simulations of bimodal trifunctional networks. ${ }^{4}$ Affine 1994.

${ }^{\otimes}$ Abstract published in Advance ACS Abstracts, November 1 transformation of mean chain end-to-end separations emerged in simulations as a consequence of the force balance at each junction, operating both in the unperturbed and in the deformed state.

Although the theoretical works of both Higgs and Ball $^{2}$ and Kloczkowski et al. ${ }^{3}$ have clarified the state of deformation for the short and the long chains at the molecular level, a clear picture of segmental orientation in bimodal networks is not yet available. On the experimental side, the FTIR measurements of Hanyu and Stein $^{5}$ and deuterium NMR measurements of Chapellier et al. ${ }^{6}$ are the only ones on segmental orientation of the two types of chains in bimodal networks. In both of these studies, the short and the long network chains are observed to undergo equal degrees of segmental orientation. Nevertheless, this interesting observation may not be considered conclusive unless it is confirmed by theory and further experiments. The purpose of the present paper is to investigate from theoretical point of view the state of segmental orientation in uniaxially deformed bimodal networks in comparison to the behavior of the corresponding unimodal networks. The theoretical approach of Kloczkowski et $\mathrm{al}^{3}{ }^{3}$ is extended to treat segmental orientation in bimodal networks. Predictions of the present paper will be used in the following paper 7 to interpret experimental data from polarized Fourier transform infrared measurements on PDMS networks.

The paper is organized as follows: In the following section, general features of three-dimensional phantom networks are briefly reviewed. Chain dimensions in undeformed phantom networks and their fluctuations are described for both unimodal and bimodal networks. In section III, the molecular deformation of unimodal and bimodal network chains is recapitulated. The extension of the theory of segmental orientation ${ }^{3}$ to bimodal networks is presented in section IV. Synergistic effects in configurational factors for segmental 
orientation, associated with the bimodality of the network, are presented in section V. Illustrative calculations are presented in section VI followed by concluding remarks in section VII.

\section{Chain Dimensions in Undeformed Phantom Networks}

The phantom network model is based on three assumptions: (i) the end-to-end separations of the network chains are Gaussianly distributed, (ii) some of the junctions at the surface of the networks are fixed and transform affinely with macroscopic strain, and (iii) the chains are subject only to topological constraints that arise directly from the connectivity of the network and to elastic forces of entropic origin. The effects of junctions and chains on one another is of no consequence. The effect of the macroscopic strain is transmitted to a chain through the junctions. This feature holds at all deformations. Mean chain dimensions $\left\langle\bar{r}^{2}\right\rangle_{0}$ in the reference state and instantaneous fluctuations $\left\langle\Delta r^{2}\right\rangle$ in chain dimensions are related to network chain dimensions $\left\langle r^{2}\right\rangle_{0}$ by

$$
\left\langle\vec{r}^{2}\right\rangle_{0}=\left(1-\frac{2}{\phi}\right)\left\langle r^{2}\right\rangle_{0}
$$

and

$$
\left\langle\Delta r^{2}\right\rangle=\frac{2}{\phi}\left\langle r^{2}\right\rangle_{0}
$$

Here an overbar refers to the time average for a given network chain, angular brackets indicate the ensemble average over all network chains, the subscript zero refers to the unperturbed state, and $\phi$ denotes the functionality of the junction.

In the case of a bimodal network, $\phi$ may be written as $\phi=\phi_{\mathrm{L}}+\phi_{\mathrm{S}}$, where $\phi_{\mathrm{L}}$ and $\phi_{\mathrm{S}}$ refer to the number of long and short chains meeting at each junction. A bimodal network in which all junctions have the same $\phi_{\mathrm{L}}$ and $\phi_{\mathrm{S}}$ is a regular bimodal network. The possibility of analytical treatment of regular bimodal networks makes them particularly attractive.

In regular bimodal networks the components referring to short and long chains, $\left\langle\bar{r}^{2}\right\rangle_{0, \mathrm{~S}}$ and $\left\langle\bar{r}^{2}\right\rangle_{0, \mathrm{~L}}$, are related to the unperturbed dimensions of the corresponding chains by ${ }^{3}$

$$
\frac{\left\langle\bar{r}^{2}\right\rangle_{0, \mathrm{~S}}}{\left\langle r^{2}\right\rangle_{0, \mathrm{~S}}}=\frac{B \xi-1}{B \xi+1}
$$

and

$$
\frac{\left\langle\bar{r}^{2}\right\rangle_{0, \mathrm{~L}}}{\left\langle r^{2}\right\rangle_{0, \mathrm{~L}}}=\frac{A-1}{A+1}
$$

where $0 \leq \xi \leq 1$ is the ratio of the contour lengths of the two types of network chains, i.e., $\xi \equiv\left\langle r^{2}\right\rangle_{0, \mathrm{~S}} /\left\langle r^{2}\right\rangle_{0, \mathrm{~L}}$. $A$ and $B$, given by Kloczkowski et al. ${ }^{3}$ are functions of the network parameters $\phi_{\mathrm{S}}, \phi_{\mathrm{L}}$, and $\xi$, thus identifying the topology of the network. $A$ and $B$ for a given network are evaluated from the numerical solution of the doublerecursion formula

$$
A=\phi_{\mathrm{L}}+\phi_{\mathrm{S}} / \xi-\frac{\phi_{\mathrm{L}}-1}{A}-\frac{\phi_{\mathrm{S}}}{B \xi^{2}}
$$

$$
B=\phi_{\mathrm{L}}+\phi_{\mathrm{S}} / \xi-\frac{\phi_{\mathrm{L}}}{A}-\frac{\phi_{\mathrm{S}}-1}{B \xi^{2}}
$$

recently derived by Kloczkowski et al. ${ }^{3}$ using Kirchhoff or valency-adjacency matrix representations of the graph theory. Likewise, fluctuations in chain dimensions are given in regular bimodal networks by the relationships ${ }^{3}$

$$
\begin{aligned}
& \frac{\left\langle(\Delta r)^{2}\right\rangle_{0, \mathrm{~S}}}{\left\langle r^{2}\right\rangle_{0, \mathrm{~S}}}=\frac{2}{B \xi+1} \\
& \frac{\left\langle(\Delta r)^{2}\right\rangle_{0, \mathrm{~L}}}{\left\langle r^{2}\right\rangle_{0, \mathrm{~L}}}=\frac{2}{A+1}
\end{aligned}
$$

Equations 3, 4, 6, and 7 hold also for the $x, y$, and $z$ components of $\mathbf{r}$.

\section{Molecular Deformation in Bimodal Phantom Networks}

Molecular deformation in networks is expressed by the tensor $\Lambda$, with the components $\Lambda_{x}{ }^{2}, \Lambda_{y}{ }^{2}$, and $\Lambda_{z}{ }^{2}$ defined as $^{9}$

$$
\begin{aligned}
& \Lambda_{x}^{2}=\frac{\left\langle x^{2}\right\rangle}{\left\langle x^{2}\right\rangle_{0}}=\frac{\left\langle\bar{x}^{2}\right\rangle+\left\langle(\Delta x)^{2}\right\rangle}{\left\langle x^{2}\right\rangle_{0}} \\
& \Lambda_{y}^{2}=\frac{\left\langle y^{2}\right\rangle}{\left\langle y^{2}\right\rangle_{0}}=\frac{\left\langle\bar{y}^{2}\right\rangle+\left\langle(\Delta y)^{2}\right\rangle}{\left\langle y^{2}\right\rangle_{0}} \\
& \Lambda_{z}^{2}=\frac{\left\langle z^{2}\right\rangle}{\left\langle z^{2}\right\rangle_{0}}=\frac{\left\langle\bar{z}^{2}\right\rangle+\left\langle(\Delta z)^{2}\right\rangle}{\left\langle z^{2}\right\rangle_{0}}
\end{aligned}
$$

where $x, y$, and $z$ refer to the components of the chain vector $\mathbf{r}$ along the axes of a laboratory-fixed frame. The second equalities in eqs 8 follow from the dot product (rrr) and from the assumption that fluctuations are independent of macroscopic deformation. For phantom networks under uniaxial stress along the $x$ direction, these components become

$$
\begin{gathered}
\Lambda_{x}{ }^{2}=(1-2 / \phi) \lambda^{2}+2 / \phi \\
\Lambda_{y}{ }^{2}=\Lambda_{z}{ }^{2}=(1-2 / \phi) \lambda^{-1}+2 / \phi
\end{gathered}
$$

Here $\lambda$ is the extension ratio along the $x$ direction, given by

$$
\lambda=\frac{\left\langle\bar{x}^{2}\right\rangle^{1 / 2}}{\left\langle\bar{x}^{2}\right\rangle_{0}^{1 / 2}}
$$

$\lambda$ represents the macroscopic deformation of the network along the stretch direction.

For bimodal networks, components of molecular deformation under uniaxial stress are obtained by substituting expressions $3,4,6$, and 7 into eq 8 as

$$
\begin{gathered}
\Lambda_{x, \mathrm{~S}}{ }^{2}=\frac{B \xi-1}{B \xi+1} \lambda^{2}+\frac{2}{B \xi+1} \\
\Lambda_{y, \mathrm{~S}}{ }^{2}=\Lambda_{z, \mathrm{~S}}{ }^{2}=\frac{B \xi-1}{B \xi+1} \lambda^{-1}+\frac{2}{B \xi+1}
\end{gathered}
$$


for short chains, and

$$
\begin{gathered}
\Lambda_{x, \mathrm{~L}}{ }^{2}=\frac{A-1}{A+1} \lambda^{2}+\frac{2}{A+1} \\
\Lambda_{y, \mathrm{~L}}{ }^{2}=\Lambda_{z, \mathrm{~L}}{ }^{2}=\frac{A-1}{A+1} \lambda^{-1}+\frac{2}{A+1}
\end{gathered}
$$

for long chains. For a unimodal network $(\xi=1, A=B$ $=\phi-1$ ), eqs 11 and 12 reduce to eq 9 . Equations 11 and 12 indicate that the long and short chains are subject to different deformations on a molecular level. When time-average chain dimensions are exclusively considered, however, the same deformation or extension ratio $\lambda$ applies to both types of chains.

\section{Segmental Orientation in Bimodal Phantom Networks}

Segmental orientation in uniaxial deformation is given by the relation ${ }^{10}$

$$
S=D^{\circ}\left(\Lambda_{x}^{2}-\Lambda_{y}^{2}\right)
$$

Here, $D^{\circ}$ is the configurational factor accounting for the effect of intrinsic structural and conformational characteristics of the network chains on segmental orientation. In the simplest treatment represented by Kuhn's freely jointed chains of $N$ equivalent bonds (or segments), $D^{\circ}$ equates to $1 / 5 N$. In more refined approaches incorporating the conformational statistics of realistic chains, a good approximation to $D^{\circ}$ is s $^{11,12}$

$$
D^{\circ}=\left(3\left\langle r^{2} \cos ^{2} \Phi\right\rangle_{0}\left\langle r^{2}\right\rangle_{0}-1\right) / 10
$$

where $\Phi$ is the angle between the end-to-end vector and the chain-embedded vectorial quantity whose orientation is being studied. The angular brackets in eq 14 refer to the ensemble average over all configurations of the network chain in the unperturbed state. Higher order moments, $\left\langle r^{2 n}\right\rangle$ and $\left\langle r^{2 n} \cos ^{2} \Phi\right\rangle$ with $n \geq 2$, appear in more rigorous expressions for $D^{\circ} .13$

Inasmuch as segmental orientation is a quantity representative of the average behavior of all network chains and observable vectorial quantities affixed along the chain contours, an expression of the form

$$
\begin{aligned}
& S(w)=w S_{\mathrm{S}}(w)+(1-w) S_{\mathrm{L}}(w)=w S_{\mathrm{S}}{ }^{\circ}+ \\
&(1-w) S_{\mathrm{L}}{ }^{\circ}+\Delta S(w)
\end{aligned}
$$

may be written for segmental orientation $S(w)$ in bimodal networks. Here, $w$ is the weight fraction of short chains in the bimodal network, which, in terms of the network structural parameters $\phi_{\mathrm{S}}, \phi_{\mathrm{L}}$, and $\xi$, reads

$$
w=\frac{\phi_{\mathrm{S}} \xi}{\phi_{\mathrm{L}}+\phi_{\mathrm{S}} \xi}
$$

$S_{\mathrm{S}}(w)$ and $S_{\mathrm{L}}(w)$ are the segmental orientations of short and long chains in the bimodal network which, themselves, depend on $w$ as indicated by the arguments, $S_{\mathrm{S}}{ }^{\circ}$ and $S_{L}{ }^{\circ}$ are the segmental orientations of the short and long chains in unimodal networks under the same topological and stretching conditions, and $\Delta S(w)$ is a perturbation term accounting for the depature from a so-called ideal behavior. As may be inferred from eq 15 , the ideal behavior refers here to the case where $\Delta \boldsymbol{S}(w)=0$. Accordingly, the segmental orientation of the bimodal network $S(w)$ is indistinguishable from the weighted sum of $S_{\mathrm{S}}{ }^{\circ}$ and $S_{\mathrm{L}}{ }^{\circ}$. This, however, does not imply that $S_{\mathrm{S}}(w)=S_{\mathrm{S}^{\circ}}$ and $S_{\mathrm{L}}(w)=S_{\mathrm{L}}{ }^{\circ}$ but, instead, a relationship of the form

$$
S_{\mathrm{L}}(w)-S_{\mathrm{L}}^{\circ}=-\left[\frac{w}{1-w}\right]\left[S_{\mathrm{S}}(w)-S_{\mathrm{S}}{ }^{\circ}\right]
$$

holds.

$\Delta S(w)$ may be viewed as a property change due to the coexistence of two chains of different lengths in the network. In geneal, it should depend on the relative amount of the two components as indicated by the argument, in analogy to the thermodynamic behavior of mixtures. For regular bimodal networks, $\Delta S(w)$ is identically equal to zero, as will be demonstrated at the end of this section.

From the combination of eqs $11-13$ one obtains for regular bimodal networks

$$
S_{\mathrm{S}}(w)=D_{\mathrm{S}}^{\circ}\left[\frac{B \xi-1}{B \xi+1}\right]\left(\lambda^{2}-\lambda^{-1}\right)
$$

and

$$
S_{\mathrm{L}}(w)=D_{\mathrm{L}} \circ\left[\frac{A-1}{A+1}\right]\left(\lambda^{2}-\lambda^{-1}\right)
$$

The terms in brackets in eqs 18 and 19 depend on network topology and composition. The dependence of $S_{\mathrm{S}}(w)$ and $\boldsymbol{S}_{\mathrm{L}}(w)$ on $w$ is implicit in the parameters $A$ and $B$, which vary with the number of short and long chains, $\phi_{\mathrm{S}}$ and $\phi_{\mathrm{L}}$, meeting at each junction in regular bimodal networks. $D_{\mathrm{L}}{ }^{\circ}$ and $D_{\mathrm{S}}{ }^{\circ}$ are functions of the long and short chain intrinsic configurational features only. They are each given by expressions analogous to eq 14 . In the simple case of the Kuhn approximation, they equate to $1 / 5 N_{\mathrm{S}}$ and $1 / 5 N_{\mathrm{L}}$, respectively, $N_{\mathrm{S}}$ and $N_{\mathrm{L}}$ referring to the number of Kuhn segments in short and long chains, $D_{\mathrm{L}}{ }^{\circ}$ and $D_{\mathrm{S}}{ }^{\circ}$ account for the molecular orientation of short or long chains in unimodal networks according to

$$
\begin{aligned}
& S_{\mathrm{S}}{ }^{\circ}=D_{\mathrm{S}}{ }^{\circ}(1-2 / \phi)\left(\lambda^{2}-\lambda^{-1}\right) \\
& S_{\mathrm{L}}{ }^{\circ}=D_{\mathrm{L}}{ }^{\circ}(1-2 / \phi)\left(\lambda^{2}-\lambda^{-1}\right)
\end{aligned}
$$

which directly follow from substitution of eqs 9 into eq 13. Equations of this form have been conveniently used in previous interpretation of segmental orientation measurements in unimodal networks. They present the advantage of relating $S$ directly to the macroscopic deformation $\lambda$. Their counterparts in bimodal networks, eqs 18 and 19 , present the same advantage.

The deviations from ideal behavior in bimodal networks may be embodied in the perturbation term $\Delta S(x)$ of eq 15 as

$$
\Delta S(w)=\Delta D(w)\left(\lambda^{2}-\lambda^{-1}\right)
$$

where $\Delta D(w)$ refers to change in configurational factor due to bimodality. For regular bimodal networks $\Delta D(w)$ is obtained by substituting eqs $18-21$ into eq 15 , as

$$
\begin{aligned}
& \Delta D(w) \equiv 2 w\left[\frac{1}{\phi}-\frac{1}{B \xi+1}\right] D_{\mathrm{S}}^{\circ}+ \\
& 2(1-w)\left[\frac{1}{\phi}-\frac{1}{A+1}\right] D_{\mathrm{L}}^{\circ}
\end{aligned}
$$

The right-hand side of eq 23 is identically zero, which 
may be shown as follows: Equation 24 may be rearranged as

$$
\frac{\phi_{\mathrm{L}} \Delta D(w)}{2(1-w) \xi D_{\mathrm{S}}{ }^{\circ}}=1-\frac{\phi_{\mathrm{S}}}{B \xi+1}-\frac{\phi_{\mathrm{L}}}{A+1}
$$

The identities $w /(1-w)=\xi \phi_{\mathrm{S}} / \phi_{\mathrm{L}}$ and $D_{\mathrm{L}}{ }^{\circ} / D_{\mathrm{S}}{ }^{\circ}=N_{\mathrm{S}} / N_{\mathrm{L}}$ $=\xi$ have been used in deriving eq 24 . The latter follows from the Kuhn approximation for the configurational factor and/or from eq 14 which has been shown to be linear in $1 / N$. Using the relation ${ }^{3}$

$$
\frac{A}{A^{2}-1}=\frac{B \xi^{2}}{B^{2} \xi^{2}-1}
$$

in eq 24 , we obtain

$$
\begin{aligned}
& \frac{\phi_{\mathrm{L}} \Delta D(w)}{2(1-w) \xi D_{\mathrm{S}}^{\circ}}= \\
& \quad \frac{B A^{2}-\left(1-\phi_{\mathrm{L}}\right) B+\phi_{\mathrm{S}} A / \xi^{2}-\left(\phi_{\mathrm{L}}+\phi_{\mathrm{S}} / \xi\right) A B}{B \xi^{4}\left(A^{2}-1\right)}
\end{aligned}
$$

The numerator of the right-hand side of eq 26 may be rewritten, by the use of eq $36 \mathrm{~b}$ of Kloczkowski et al., ${ }^{3}$ as $B A^{2}-A B^{2}-B+A / \xi^{2}$, which equates to zero upon substitution of eq $36 \mathrm{a}$ of the same reference. Thus, we arrive at the important conclusion that $\Delta D(w)=0$ and consequently $\Delta S(w)=0$ for regular bimodal networks.

It is noted that substitution of eqs 6 and 7 into eq 24 and using $\Delta D(w)=0$ lead to the following relation among the fluctuations of long and short chains:

$$
\frac{\phi_{\mathrm{S}}\left\langle\left(\Delta r_{\mathrm{S}}\right)^{2}\right\rangle}{\left\langle r_{\mathrm{S}}{ }^{2}\right\rangle_{0}}+\frac{\phi_{\mathrm{L}}\left\langle\left(\Delta r_{\mathrm{L}}\right)^{2}\right\rangle}{\left\langle r_{\mathrm{L}}{ }^{2}\right\rangle_{0}}=2
$$

This reduces to the well-known expression

$$
\frac{\left\langle(\Delta r)^{2}\right\rangle}{\left\langle r^{2}\right\rangle_{0}}=\frac{2}{\phi}
$$

for unimodal networks.

As mentioned above, the fact that $\Delta S(w)=0$ for regular bimodal networks does not exclude the possibility of perturbations in the segmental orientation of short and long chains in bimodal networks. On the contrary, in bimodal networks the segmental orientation of short and long chains is observed to differ from those taking place in unimodal networks. Qualitatively, a decrease in orientation is observed ${ }^{6,7}$ in short chains due to the presence of long chains, and an increase in orientation of long chains is imparted by the presence of short chains in bimodal networks, as intuitively expected. The following paper ${ }^{7}$ will concentrate in those changes in segmental orientations of individual chains due to bimodality of the network.

\section{v. Changes in Configurational Factors of Individual Chains}

Equations 18 and 19 consist of the product of three terms accounting for (i) the effect of intrinsic configurational factor through $D_{\mathrm{S}}{ }^{\circ}$ or $D_{\mathrm{L}}{ }^{\circ}$, (ii) the network topology and composition, and (iii) the macroscopic strain. From the examination of eqs 18-21, we observe that the changes in the segmental orientations of the network chains in bimodal networks relative to those occurring in unimodal networks, arise from the changes in the second term, which reflects the effect of network topology and composition. Mainly, the terms in brackets in eqs 18 and 19 reduce to the term $(1-2 / \phi)$ in unimodal networks. For convenience, we define the product of the two contributions (i) and (ii) as the effective configurational factors. Using eqs 18-21, the changes $\Delta S_{S}(w)$ and $\Delta S_{\mathrm{L}}(w)$ in the segmental orientations of short and long chains, respectively, in regular bimodal networks read

$$
\begin{aligned}
& \Delta S_{\mathrm{S}}(w) \equiv S_{\mathrm{S}}(w)-S_{\mathrm{S}}^{\circ}=\Delta D_{\mathrm{S}}(w)\left(\lambda^{2}-\lambda^{-1}\right) \\
& \Delta S_{\mathrm{L}}(w) \equiv S_{\mathrm{L}}(w)-S_{\mathrm{L}}^{\circ}=\Delta D_{\mathrm{L}}(w)\left(\lambda^{2}-\lambda^{-1}\right)
\end{aligned}
$$

where

$$
\Delta D_{\mathrm{S}}(w)=2\left[\frac{1}{\phi}-\frac{1}{B \xi+1}\right] D_{\mathrm{S}}^{\circ}
$$

and

$$
\Delta D_{\mathrm{L}}(w)=2\left[\frac{1}{\phi}-\frac{1}{A+1}\right] D_{\mathrm{L}}^{\circ}
$$

are the changes in the effective configurational factors of short and long chains, respectively, due to the bimodality of the network. The dependence of $\Delta D_{\mathrm{S}}(w)$ and $\Delta D_{\mathrm{L}}(w)$ on the composition of the network arises from the dependence of the parameters $A$ and $B$ on network topology, as already mentioned for $\Delta S_{\mathrm{S}}(w)$ and $\Delta S_{\mathrm{L}}(w)$. The dependence of $\Delta D_{\mathrm{S}}(w)$ and $\Delta D_{\mathrm{L}}(w)$ on network composition will be illustrated in the next section.

It is interesting to note that, in regular bimodal networks, which were shown above to exhibit the ideal behavior, defined as $\Delta S(w)=\Delta D(w)=0$, the changes in the configurational factors of short and long chains, given by eqs 31 and 32 , will have opposite sign and will be related to each other by an expression equivalent to eq 17 , i.e.,

$$
\Delta D_{\mathrm{L}}(w)=-\frac{w}{1-w} \Delta D_{\mathrm{S}}(w)
$$

This equation readily follows from the substitution of eqs 29 and 30 into eq 17. The use of eqs 31 and 32 in eq 33 yields

$$
D_{\mathrm{L}}{ }^{\circ}=-\frac{w}{1-w}\left[\frac{1}{\phi}-\frac{1}{B \xi+1}\right]\left[\frac{1}{\phi}-\frac{1}{A+1}\right]^{-1} D_{\mathrm{S}}^{\circ}
$$

Equation 34 reduces to eq 27 upon substitution of $D_{\mathrm{L}} \%$ $D_{\mathrm{S}}{ }^{\circ}=\xi$ and using eq 16 .

\section{Illustrative Calculations}

The changes in configurational factors of short and long chains induced by the bimodality of the network are shown in Figures 1 and 2 as a function of the ratio $\xi=\left\langle r^{2}\right\rangle_{0, \mathrm{~S}} \delta\left\langle r^{2}\right\rangle_{0, \mathrm{~L}}$ of the unperturbed mean-square lengths of short and long chains. The curves in Figure 1 are obtained for the three possible types of regular bimodal networks with functionality $\phi=4$, mainly $\phi_{\mathrm{S}}=\phi-\phi_{\mathrm{I}}$ $=1,2$, and 3 . These are indicated by the labels $S_{1} L_{3}$, $\mathrm{S}_{2} \mathrm{~L}_{2}$, and $\mathrm{S}_{3} \mathrm{~L}_{1}$, respectively. Those in Figure 2 are obtained for trifunctional regular bimodal networks for the two cases $S_{1} L_{2}$ and $S_{2} L_{1}$. The upper curves in both figures represent the ratio $\Delta D_{\mathrm{L}} / D_{\mathrm{L}}{ }^{\circ}$ corresponding to the 


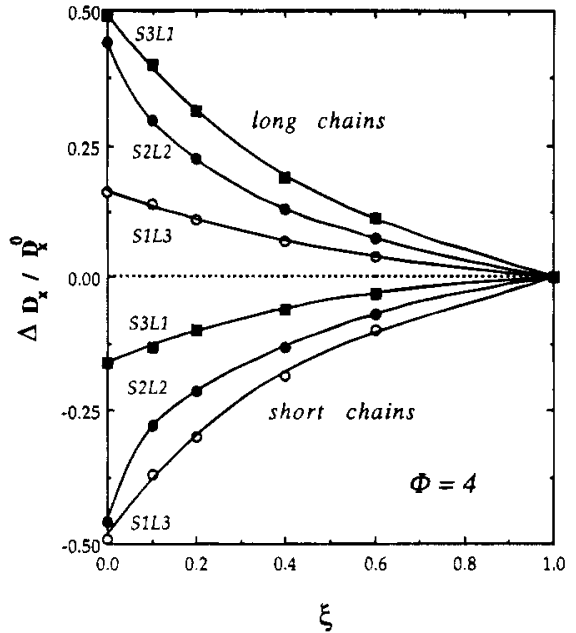

Figure 1. Change in configurational factors $\Delta D_{\mathrm{x}} / D_{\mathrm{x}}{ }^{\circ}$ of short $(\mathrm{x}=\mathrm{S})$ and long $(\mathrm{x}=\mathrm{L})$ chains induced by bimodality in regular tetrafunctional networks. The upper curves in both Figures 1 and 2 represent the ratio $\Delta D_{\mathrm{L}} / D_{\mathrm{L}}{ }^{\circ}$ corresponding to the long chains, while the lower three curves display the ratio $\Delta D_{\mathrm{S}} / D_{\mathrm{S}}{ }^{\circ}$ for short chains. The points indicate the results from calculations using eqs 31 and 32 . Best fitting curves through these results are drawn to guide the eye.

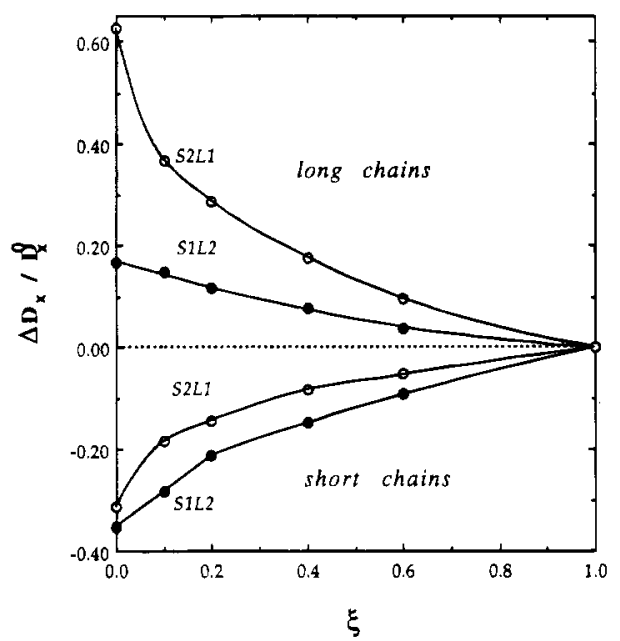

Figure 2. Change in configurational factors $\Delta D_{\mathrm{x}} / D_{\mathrm{x}}^{\circ}$ of short $(x=S)$ and long $(x=L)$ chains induced by bimodality in regular trifunctional networks. See legend for Figure 1.

long chains, while the lower curves display the ratio $\Delta D_{\mathrm{S}} / D_{\mathrm{S}}{ }^{\circ}$ for short chains. The points indicate the results from calculations using eqs 31 and 32 . Best fitting curves through these results are drawn to guide the eye.

The long chains exhibit a positive deviation from their values in the unimodal network, whereas a negative departure is observed in short chains. Inasmuch as the increase in the value of the configurational factors is directly reflected upon the segmental orientation, as delineated in eqs 29 and 30 , these results indicate that the long chains are more oriented in bimodal networks compared to chains of the same length in unimodal networks. The short chains, on the other hand, exhibit a decrease in the configurational factor, which leads to weaker orientation of short chains in bimodal networks compared to the behavior of their unimodal counterparts.

The ratios $S_{\mathrm{S}} / S_{\mathrm{S}}{ }^{\circ}$ and $S_{\mathrm{I}} / S_{\mathrm{L}}{ }^{\circ}$ of the segmental orientations of short and long chains in bimodal networks compared to those observed in unimodal networks subject to the same functionality and deformation are

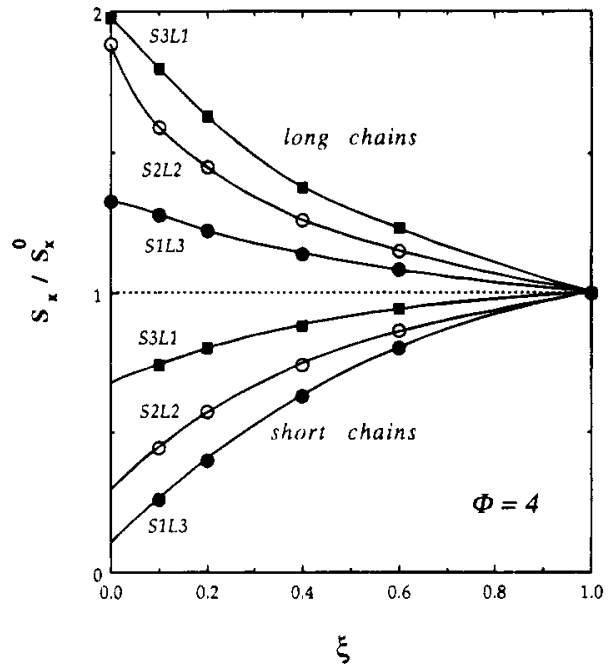

Figure 3. Ratio $S_{\mathrm{x}} / S_{\mathrm{x}}{ }^{\circ}$ of the segmental orientations of short $(x=S)$ and long $(x=L)$ chains in bimodal networks compared to those observed in unimodal networks subject to the same functionality and deformation in tetrafunctional regular networks calculated from eqs 35 and 36 . These results hold irrespective of the state of deformation and intrinsic chain configurational characteristics.

shown in Figure 3 for tetrafunctional regular networks. These are calculated from the equations

$$
\begin{gathered}
\frac{S_{\mathrm{S}}}{S_{\mathrm{S}}{ }^{\circ}}=(1-2 / \phi)^{-1}\left[\frac{B \xi-1}{B \xi+1}\right] \\
\frac{S_{\mathrm{L}}}{S_{\mathrm{L}}{ }^{\circ}}=(1-2 / \phi)^{-1}\left[\frac{A-1}{A+1}\right]
\end{gathered}
$$

which readily follow from the ratio of eqs 18 to 20 and eq 19 to 21 . The extension ratio being eliminated in this ratio, the curves are independent of macroscopic deformation. Yet, it should be recalled that eqs 18-21 represent the first-order approximation for segmental orientation in which higher order terms in $1 / \mathrm{N}$ are neglected. Thus, the curves in Figure 3 are applicable, in a strict sense, for networks of sufficiently long chains, which are subject to relatively small deformations.

In analogy to Figures 1 and 2, the curves above the horizontal line $S / S^{\circ}=1$ in Figure 3 represent the ratio $S_{\mathrm{I}} / S_{\mathrm{L}}{ }^{\circ}$ of long chains; the lower curves display $S_{\mathrm{S}} / S_{\mathrm{S}}{ }^{\circ}$. The short chains in bimodal networks with $\phi_{\mathrm{L}}=1$ (i.e., $\mathrm{S}_{3} \mathrm{~L}_{1}$ ) are weakly affected by the presence of one long chain at each junction. Likewise, the long chains in bimodal networks of type $S_{1} L_{3}$ exhibit small deviations from their unimodal counterparts. Therefore the introduction of a low number of chains of a different length in a unimodal network has a weak effect on the orientation behavior of the original chains. The departures from unimodal network behavior for chains of a given length, either $\mathrm{S}$ or $\mathrm{L}$, become more significant if those chains are in lower proportion in the bimodal network. Thus, in the case of three long chains at a given junction, the segmental orientation of short chains in bimodal networks strongly departs from that observed in unimodal networks, coming closer to the isotropic value $S=0$ as the ratio $\xi$ decreases. The orientation of the long chains, on the other hand, is systematically increased by increasing amount of short chains. It is enhanced by a factor of 2 in the limit of $\xi$ $=0$ for bimodal networks of the type $\mathrm{S}_{3} \mathrm{~L}_{1}$. We also note the symmetric shape of the curves for short and long chains with respect to the horizontal line $S / S^{\circ}=1$. 


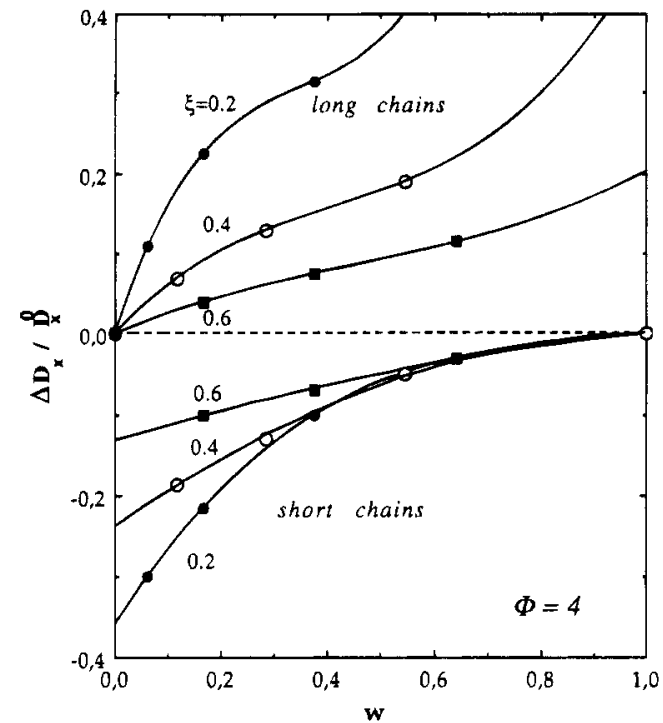

Figure 4. Changes in effective configurational factors $\Delta D_{\mathrm{L}}(w)$ and $\Delta D_{\mathrm{S}}(w)$ as a function of the weight fraction, $w$, of short chains in the network, for regular tetrafunctional networks with various ratios $\xi$, as indicated by the labels. The curves through the points are the best fitting third-order polynomials.

Figure 4 displays the changes in effective configurational factors $\Delta D_{\mathrm{L}}(w)$ and $\Delta D_{\mathrm{S}}(w)$ as a function of the weight function of short chains in the network. The curves are drawn for regular tetrafunctional networks using eqs 31 and 32 . Various ratios $\xi$, as indicated by the labels, are selected for illustrative purposes, and the corresponding changes $\Delta D_{\mathrm{L}}(w) / D_{\mathrm{L}}{ }^{\circ}$ and $\Delta D_{\mathrm{S}}(w) / D_{\mathrm{S}}{ }^{\circ}$ are determined using Figure 1. Thus, values for three points corresponding to the networks $\mathrm{S}_{1} \mathrm{~L}_{3}, \mathrm{~S}_{2} \mathrm{~L}_{2}$, and $\mathrm{S}_{3} \mathrm{~L}_{1}$ are calculated for each $\xi$, apart from the limiting values $\Delta D_{\mathrm{L}}(0) / D_{\mathrm{L}}{ }^{\circ}=0$ and $\Delta D_{\mathrm{S}}(1) / D_{\mathrm{S}}{ }^{\circ}=0$. The curves through the points are the best fitting third-order polynomials. It is noted that the relative changes in the configurational factors of the long chains are positive, indicating an enhancement in orientation, due to the presence of short networks, whereas the short chains are less sensitive to the presence of long chains and exhibit a decrease in the effective configurational factor which leads to a weakening in preferential alignment.

\section{Conclusion}

The present study has extended the recently developed $^{3}$ theoretical analysis of regular bimodal networks to treat the segmental orientation of the two types of chains as a function of the configurational characteristics of the chains, network topology and composition, and macroscopic state of deformation. Results of the formulation show that segmental orientation in the long chains of the network is larger than the corresponding one in the unimodal network. Conversely, shorter chains orient less in the bimodal network compared to the corresponding chains in the unimodal network. These predictions will be tested against experimental data from Fourier transform infrared study of wellcharacterized PDMS networks presented in the following paper.

Acknowledgment. Partial support from Bogazici University Research Funds Project 93P0084 is gratefully acknowledged.

\section{References and Notes}

(1) Mark, J. E.; Erman, B. Rubberlike Elasticity: A Molecular Primer; Wiley-Interscience: New York, 1988; p 196.

(2) Higgs, P. G.; Ball, R. C. J. Phys. (Paris) 1988, 49, 1785.

(3) Kloczkowski, A.; Mark, J. E.; Erman, B. Macromolecules $1991,24,3266$.

(4) Sakrak, G.; Bahar, I.; Erman, B. Makromol. Chem.: Theory Simul. 1994, 3, 151.

(5) Hanyu, A.; Stein, R. S. Makromol. Chem., Macromol. Symp. $1991,45,189$.

(6) Chapellier, B.; Deloche, B.; Oeser, R. J. Phys. II Fr. 1993, 3, 1619 .

(7) Besbes, S.; Bokobza, L.; Monnerie, L.; Bahar, I.; Erman, B. Macromolecules 1995, 28, 231.

(8) Flory, P. J. Proc. R. Soc. London A 1976, 351, 351

(9) Erman, B.; Flory, P. J. Macromolecules 1983, 16, 1601, 1607.

(10) Erman, B.; Monnerie, L. Macromolecules 1985, 18, 1985.

(11) Nagai, K. J. Chem. Phys. 1964, 40, 2818.

(12) Erman, B.; Bahar, I. Macromolecules 1988, 21, 452.

(13) Erman, B.; Haliloglu, T.; Bahar, I.; Mark, J. E. Macromolecules 1991, 24, 901. 